\title{
Genome-wide identification of MAPK gene family members in Fagopyrum tataricum and their expression during development and stress responses
}

\author{
Yingjun Yao ${ }^{\dagger}$, Haixia Zhao ${ }^{\dagger}$, Lei Sun, Wenjing Wu, Chenglei Li and Qi Wu*
}

\begin{abstract}
Background: Mitogen-activated protein kinases (MAPKs) plays essential roles in the development, hormone regulation and abiotic stress response of plants. Nevertheless, a comprehensive study on MAPK family members has thus far not been performed in Tartary buckwheat.

Results: Here, we identified 16 FtMAPKs in the Fagopyrum tataricum genome. Phylogenetic analysis showed that the FtMAPK family members could be classified into Groups A, B, C and D, in which A, B and C members contain a Thr-GluTyr (TEY) signature motif and Group D members contain a Thr-Asp-Tyr (TDY) signature motif. Promoter cis-acting elements showed that most Pro FtMAPks contain light response elements, hormone response elements and abiotic stress response elements, and several Pro FtMAPks have MYB-binding sites, which may be involved in the regulation of flavonoid biosynthesis-related enzyme gene expression. Synteny analysis indicated that FtMAPKs have a variety of biological functions. Protein interaction prediction suggested that MAPKs can interact with proteins involved in development and stress resistance. Correlation analysis further confirmed that most of the FtMAPK genes and transcription factors involved in the stress response have the same expression pattern. The transient transformation of FtMAPK1 significantly increased the antioxidant enzymes activity in Tartary buckwheat leaves. In addition, we also found that FtMAPK1 can respond to salt stress by up-regulating the transcription abundance of downstream genes.

Conclusions: A total of 16 MAPKs were identified in Tartary buckwheat, and the members of the MAPK family containing the TDY motif were found to have expanded. The same subfamily members have relatively conserved gene structures and similar protein motifs. Tissue-specific expression indicated that the expression of all FtMAPK genes varied widely in the roots, stems, leaves and flowers. Most FtMAPKs can regulate the expression of other transcription factors and participate in the abiotic stress response. Our findings comprehensively revealed the FTMAPK gene family and laid a theoretical foundation for the functional characterization of FtMAPKs.
\end{abstract}

Keywords: Tartary buckwheat, MAPK, Evolution, Expression patterns, Abiotic stress, Functional verification

*Correspondence: wuqi@sicau.edu.cn

${ }^{\dagger}$ Yingjun Yao and Haixia Zhao contributed equally to this work.

College of Life Science, Sichuan Agricultural University, No.46, Xinkang

Road, Ya'an 625014, Sichuan, China

\section{Background}

To respond to various biotic and abiotic threats, plants have evolved a series of sophisticated signalling networks to manage external stresses. MAPK cascades play critical roles in the response to external stimuli and represent one of the primary mechanisms controlling signal transduction. The MAPK cascade pathway 
transmits external stimulus signals to cells, after which the organism realizes the regulation of response to the stimulus through the phosphorylation and dephosphorylation of proteins.

MAPK signal transduction modules are traditionally composed of three sequentially activated kinases. In general, external signals can activate MAPKKKs, which phosphorylate downstream MAPKKs, while phosphorylated MAPKKs ultimately phosphorylate MAPKs [1]. Activated MAPKs eventually phosphorylate various downstream transcription factors and other signalling components [2].

MAPKs function towards the end MAPK cascade pathways and contain 11 conserved protein kinase motifs. There is a TXY motif between domains VII and VII [3], and phosphorylated TXY is essential for activating MAPK. Plant MAPKs can be separated into Groups A, B, C, and D. Members of Groups A, B, and C contain the characteristic Thr-Glu-Tyr (TEY) motif at their phosphorylation site. However, members of Group D possess a Thr-Asp-Tyr (TDY) motif. Due to the importance of MAPKs in coping with adverse conditions, the MAPK gene family has been studied in depth: members of this family have been identified and reported in various plant species and present quite complex features. To date, there are 20,15, 19, 19, 18, 20, 28, and 54 MAPKs in Arabidopsis [2], rice [4], maize [5], chickpea [6], Actinidia chinensis [7], barley [8], Gossypium raimondii [9] and bread wheat [10]. Similarly, 12 candidate MAPK genes have been identified in Fragaria vesca [11]. With an improved in-depth understanding of MAPK, the function of $M A P K$ has attracted widespread attention and research.

A large number of studies have confirmed that MAPKs participate in a variety of biological functions. The expression levels of all MAPKs are increased significantly under abiotic stress and abscisic acid (ABA) treatment in chickpea [6]. Overexpression of OsMAPK33 weakened the tolerance to salt stress in rice [12]. Under cold treatment, the expression of MAPKs increased significantly in Jatropha curcas. MAPKs are also involved in the growth and development of plants. AtMAPK6 kinase is involved in regulating many aspects of plant development, including the development of anthers, inflorescences, and embryos [13]. In addition, $M A P K s$ are involved in response to hormones. Specifically, several MAPKs are involved in the ethylene response process during the ripening of bananas. Moreover, MAPK can interact with other transcription factors in response to adversity. OsWRKY55 interacts with four MAPKs that could be induced by drought: OsMPK7, OsMPK9, OsMPK201, and OsMPK20-4 [14]. MdMYB1 can be phosphorylated by MdMPK4 to enhance light-induced anthocyanin accumulation in apples [15]. Therefore, MAPKs confer essential biological functions directly or in combination with transcription factors to plants.

Tartary buckwheat, a grain crop species used for both food and medicine, has attracted much attention because of its rich content of flavonoids. Because Tartary buckwheat has good adaptability to drought, salt, high temperature/low temperature; etc., it is crucial to reveal stress resistance mechanisms and identify stress resistance-related gene families. The MAPK cascade is an essential aspect of studying the mechanism of resistance to stress in plants. We conducted comprehensive research, including a phylogenetic, chromosome localization, gene structure, protein motif, cis-acting element, evolutionary, expression profile, protein interaction, correlation analysis and functional verification. Overall, a comprehensive study of the FtMAPK gene family and the expression pattern of FtMAPK in response to abiotic stress have laid a foundation for the functional characterization and expression regulation of FtMAPK in Tartary buckwheat.

\section{Results}

Identification of MAPK family genes in Tartary Buckwheat

To fully understand the evolutionary history of the MAPK family of Tartary buckwheat and its importance in abiotic stress, a total of 16 MAPK genes were identified in Tartary buckwheat, designated FtMAPK1-FtMAPK16 (Additional file 1). All MAPKs in Tartary buckwheat contained the phosphorylation sites TEY or TDY by Batch-CD Search (https://www.ncbi.nlm.nih.gov/Struc ture/bwrpsb/bwrpsb.cgi) analysis and TBtools visualization (Additional file 2).

In addition, we analysed the characteristics of the 16 FtMAPKs in Fagopyrum tataricum (Table 1). Briefly, the protein length ranged from 371(MAPK6) to 609 (MAPK16) amino acids. The protein molecular weight (MW) ranged from $42.6 \mathrm{kDa}$ (MAPK6) to $69.4 \mathrm{kDa}$ (MAPK16), and the isoelectric point (pI) varied from 5.39 (MAPK4) to 9.25 (MAPK11). Subcellular localization prediction revealed that 8 MAPK proteins were located in the cytoplasm, 3 MAPK proteins were located in the microbody, 1 MAPK protein was found in the chloroplast stroma, and the other proteins were located in the nucleus. However, none of the MAPK proteins have a transmembrane domain. Only two MAPK proteins (MAPK4 and MAPK14) have signal peptides, and none of the other MAPK proteins have signal peptides (Table 1).

\section{Phylogenetic analyses of FtMAPK genes}

To investigate the evolutionary relationships among the MAPK proteins, a phylogenetic tree was constructed with amino acid sequences of 16 putative FtMAPKs 
Table 1 Characteristics of 16 the FtMAPKs in Tartary buckwheat

\begin{tabular}{|c|c|c|c|c|c|c|c|}
\hline Gene name & Gene ID & $\begin{array}{l}\text { Protein length } \\
\text { (aa) }\end{array}$ & MW & pl & $\begin{array}{l}\text { Subcellular } \\
\text { location }\end{array}$ & $\begin{array}{l}\text { Transmembrane } \\
\text { domain }\end{array}$ & Signal peptide \\
\hline FtMAPK1 & FtPinG0006545900.01 & 385 & 44.2 & 6.86 & M & 0 & NO \\
\hline FtMAPK2 & FtPinG0004152000.01 & 388 & 44.2 & 6.19 & $\mathrm{~N}$ & 0 & NO \\
\hline FtMAPK3 & FtPinG0005197700.01 & 407 & 46.7 & 5.43 & C & 0 & NO \\
\hline FtMAPK4 & FtPinG0000626000.01 & 372 & 43.0 & 5.39 & N & 0 & YES \\
\hline FtMAPK5 & FtPinG0004095200.01 & 406 & 46.4 & 6.90 & CS & 0 & NO \\
\hline FtMAPK6 & FtPinG0001815800.01 & 371 & 42.6 & 5.41 & C & 0 & NO \\
\hline FtMAPK7 & FtPinG0006454600.01 & 381 & 44.3 & 5.66 & C & 0 & NO \\
\hline FtMAPK8 & FtPinG0008104600.01 & 452 & 51.2 & 8.34 & C & 0 & NO \\
\hline FtMAPK9 & FtPinG0005315200.01 & 567 & 64.7 & 8.84 & N & 0 & NO \\
\hline FtMAPK10 & FtPinG0009258900.01 & 608 & 68.9 & 9.40 & C & 0 & NO \\
\hline FtMAPK11 & FtPinG0005630300.01 & 589 & 66.9 & 9.25 & C & 0 & NO \\
\hline FtMAPK12 & FtPinG0005324100.01 & 405 & 47.1 & 8.93 & M & 0 & NO \\
\hline FtMAPK13 & FtPinG0003584300.01 & 570 & 64.6 & 9.33 & M & 0 & NO \\
\hline FtMAPK14 & FtPinG0009311600.01 & 543 & 61.8 & 6.51 & C & 0 & YES \\
\hline FtMAPK15 & FtPinG0009346800.01 & 571 & 65.5 & 7.41 & C & 0 & NO \\
\hline FtMAPK16 & FtPinG0001873100.01 & 609 & 69.4 & 9.47 & $\mathrm{~N}$ & 0 & $\mathrm{NO}$ \\
\hline
\end{tabular}

MW molecular weight, $p /$ isoelectric points, $M$ microbody, $N$ nucleus, $C$ cytoplasm, $C S$ chloroplast stroma

from Tartary buckwheat, 20 AtMAPKs from Arabidopsis, 15 OsMAPKs from Oryza sativa and 28 GrMAPKs from Gossypium raimondii (Fig. 1). The results showed that the 16 FtMAPKs could be divided into Groups A, B, $\mathrm{C}$ and D. Among them, FtMAPKs belonging to Groups $\mathrm{A}, \mathrm{B}$, and $\mathrm{C}$ have a TEY motif, whereas FtMAPKs classified into Group D possess a TDY motif. FtMAPK3 and FtMAPK7 were assigned to Cluster A; FtMAPK2, FtMAPK4, FtMAPK5 and FtMAPK6 were assigned to Cluster B; and FtMAPK1 clustered into Cluster C. However, FtMAPK8, FtMAPK9, FtMAPK10, FtMAPK11, FtMAPK12, FtMAPK13, FtMAPK14, FtMAPK15, and FtMAPK16 were assigned to Cluster D (Fig. 1). Interestingly, the FtMAPKs in Group D had significantly expanded, as 9 FtMAPKs were present in Tartary buckwheat. Moreover, the other 3 species also had the most members in Group D, and the expansion of Group D genes provides a reference for the evolution of Tartary buckwheat. Compared with group D, Group C included only FtMAPK1, indicating that the members of Group $\mathrm{C}$ had significantly contracted. We speculate that FtMAPK1 is very important for the study of the function of Tartary buckwheat.

\section{Chromosomal location of FtMAPKs in the Tartary buckwheat genome}

Chromosome mapping analysis revealed that 16 FtMAPK genes were present on all chromosomes except chromosome 6. The results showed that chromosomes Ft2 and Ft3 have only one FtMAPK, FtMAPK7 and FtMAPK5, respectively. In addition, two FtMAPK genes were found on chromosomes $\mathrm{Ft} 4, \mathrm{Ft} 5$, and $\mathrm{Ft} 8$. In contrast, half of the FtMAPK genes were distributed on chromosomes Ft1 and Ft7 (Fig. 2). Furthermore, FtMAPK presents an uneven distribution, and no tandem repeat genes appear. Remarkably, FtMAPKs belonging to the same group in the phylogenetic tree are not mapped on the same chromosome, but are scattered in different positions on the same chromosome, or exist on different chromosomes. This shows that tandem duplication events did not play an important role during the expansion of the FtMAPK family.

\section{Gene structure and motif composition of MAPKs in Tartary buckwheat}

A phylogenetic tree was constructed from 16 amino acid sequences of FtMAPK, and the gene family was divided into four groups. Group D is the largest with 9 members, but group $\mathrm{C}$ has only one member FtMAPK1 (Fig. 3A). We analysed the conserved motifs of MAPKs to explore the various biological functions of the protein domains. Fifteen conserved motifs were identified, and each motif is present (Figs. 3B and D). The 16 identified MAPK proteins contained motif 2 (which contains the TDY signature motif) or motif 8 (which contains the TEY signature motif), indicating that all FtMAPKs belong to the $M A P K$ gene family. Specifically, the members in the same subfamily shared similar conserved motifs. Nine MAPKs belonging to Group D contained TDY characteristic motifs, and the MAPKs classified into Groups A, B 


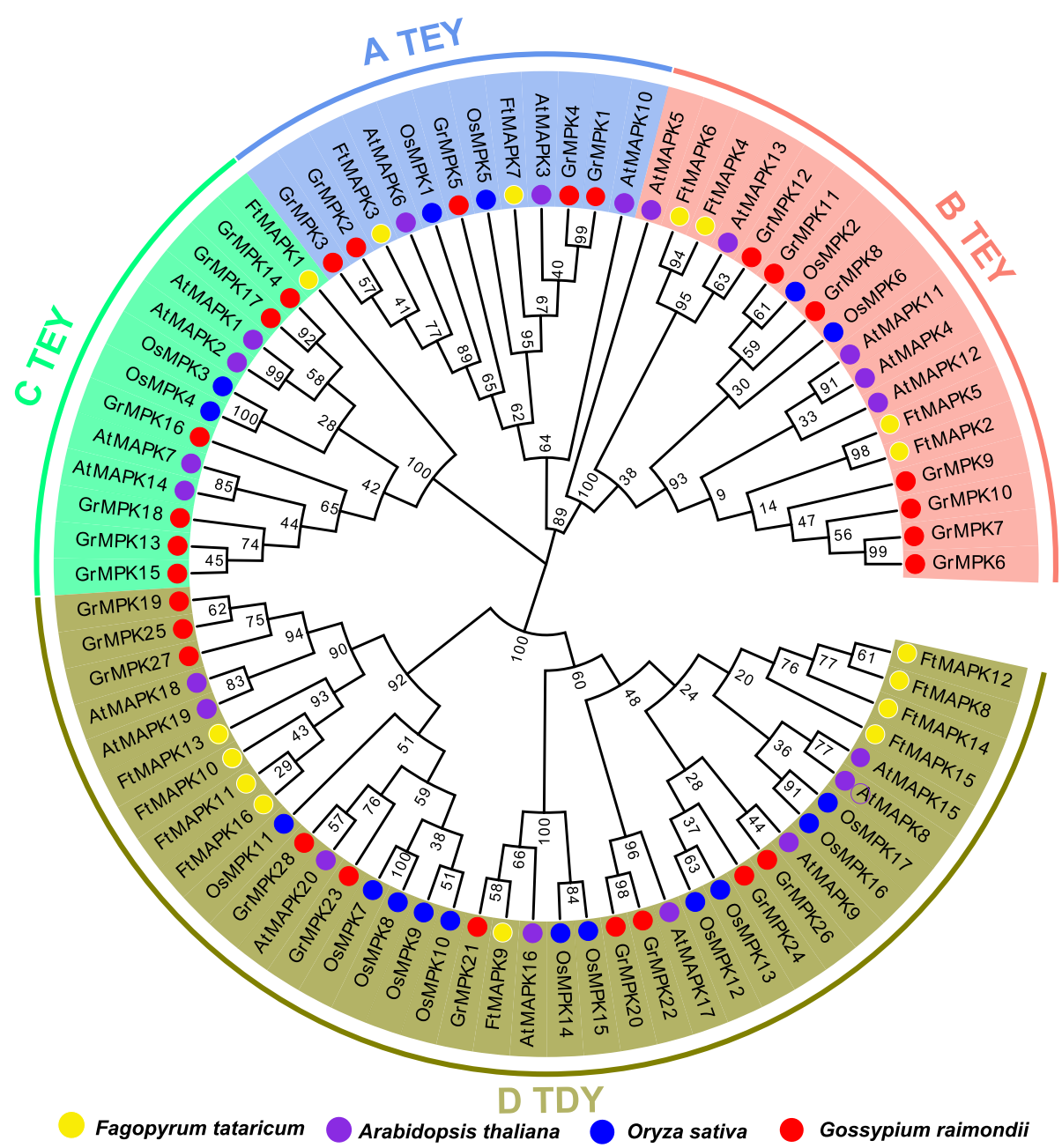

Fig. 1 Phylogenetic relationship of MAPK genes in F. tataricum, Arabidopsis, O.sativa and G.raimondii. A TEY, B TEY, C TEY and D TDY exhibit different gene clusters. The full-length MAPK protein sequences were aligned using the MUSCLE tool, and the phylogenetic tree was constructed using MEGA 7.0.26 by the NJ method with default parameters and 1000 bootstrap replicates. Finally, the tree was visualized with the online tool EvolView (https://evolgenius.info//evolview-v2/\#mytrees/1/2)

and $\mathrm{C}$ contained characteristic TEY motifs. N-terminal motif 9 of MAPK in Group D also contains the TDY/ TEY motif, but it is not particularly conserved. Additionally, the FtMAPK of Group D includes specific motif 9 at the N-terminus and specific motifs 7, 13, 14 and 15 at the $\mathrm{C}$-terminus. Whether the differences in motif composition between the members of Group D and the members of Groups A, B and C affect their biological functions, needs to be further investigated through biological experiments (Fig. 3B).

We also investigated the exon-intron pattern of the 16 FtMAPKs (Fig. 3C). The results showed that FtMAPKs in Group A exhibit conservation of their exon-intron distribution. The two FtMAPKs with the closest relationship in Group B had the same intron-exon distribution pattern.
However, FtMAPK1 in Group C includes only two exonsintrons. The structures of FtMAPKs in Groups A, B and $\mathrm{C}$ were more conserved than were those of the FtMAPKs in Group D, showing a complex distribution of exons and introns. FtMAPK12 has seven exons, FtMAPK8, FtMAPK9, FtMAPK14 and FtMAPK15 have 11 exons, and the other FtMAPKs consist of 13 exons.

\section{Analysis of FtMAPKs promoter}

To better understand the function of FtMAPKs and the precise regulation of stress-responsive gene expression, the cis-acting elements in the promoter region of FtMAPKs were identified and analysed (Fig. 4; Additional file 3). The results suggested that most FtMAPKs contain hormone (methyl jasmonate [MeJA] and ABA) 


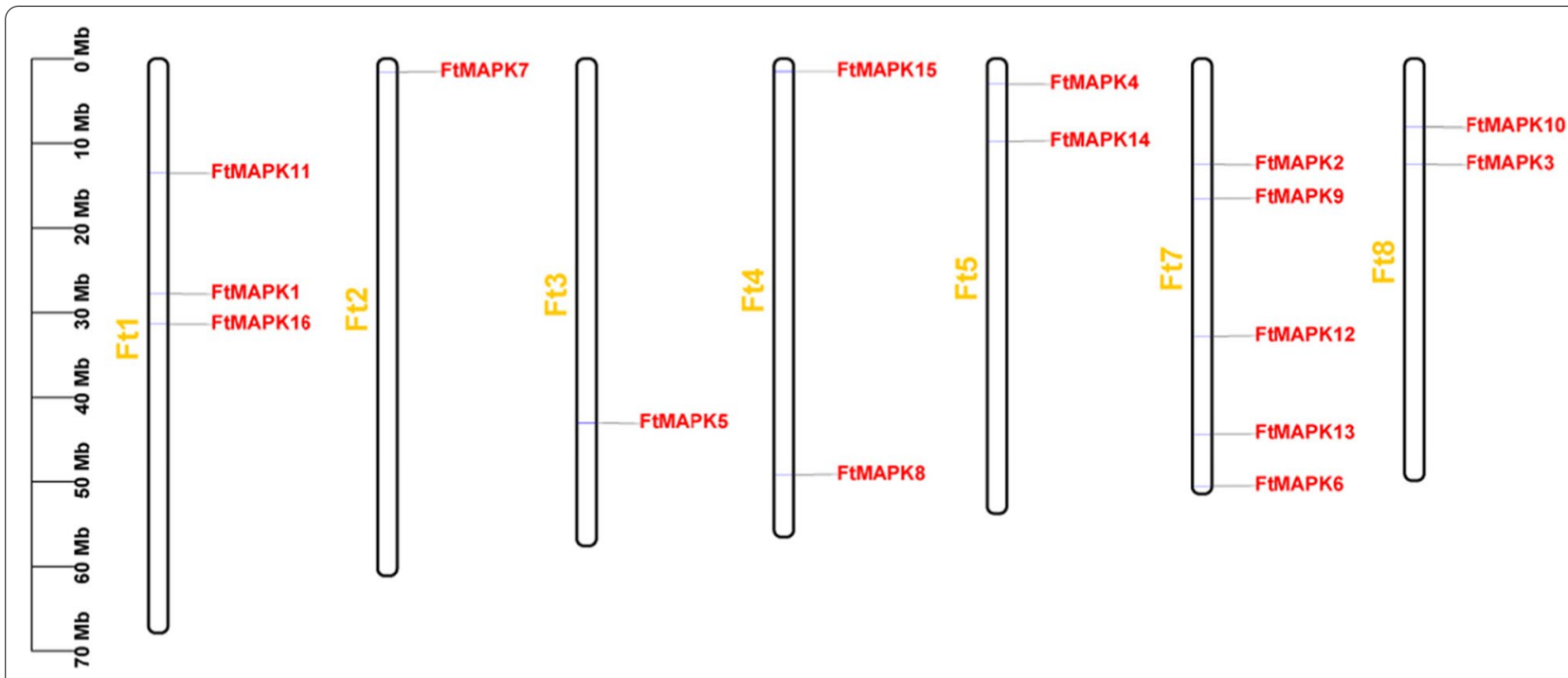

Fig. 2 Schematic representations of the chromosomal distributions of the FtMAPK genes. The vertical bars mark the chromosomes of Tartary buckwheat. The chromosome number is located to the left of each chromosome. The scale on the left represents the chromosome length

responsive elements. All Pro ${ }_{F t M A P K s}$ contain light-responsive elements. The promoters of 7 FtMAPKs (FtMAPK1, FtMAPK3, FtMAPK9, FtMAPK11, FtMAPK12, FtMAPK14 and FtMAPK15) contain regulatory elements (O2 sites) involved in zein metabolism regulation. A few FtMAPKs have CAT boxes and circadian function engaged in endosperm expression and circadian control, respectively. The cis-acting elements in the promoters of other FtMAPKs are also involved in the abiotic stress response, including 6 Pro $_{\text {FtMAPKs }}$ (FtMAPK6, FtMAPK7, FtMAPK10, FtMAPK13, FtMAPK14 and FtMAPK15) containing cis-regulating elements involved in defence and stress responsiveness, and 9 Pro $_{\text {FtMAPKs }}$ (FtMAPK2, FtMAPK3, FtMAPK4, FtMAPK6, FtMAPK9, FtMAPK10, FtMAPK11, FtMAPK14 and FtMAPK16) containing cisregulating elements involved in low-temperature responsiveness. There are even several Pro FtMAPKs $_{\text {(FtMAPK2, }}$ FtMAPK4, FtMAPK9, FtMAPK12 and FtMAPK16) that combine with MYB transcription factors to participate in the drought response. In addition, there are three Pro $_{F t-}$ MAPKs (FtMAPK3, FtMAPK10 and FtMAPK16) that can be combined with MYB to participate in the regulation of flavonoid biosynthesis. There are also cis-regulatory elements involved in the expression of meristems (Fig. 4). These results illustrate the potential role of FtMAPKs in plant growth and development, the response to stress and hormone signalling pathways.

\section{Tertiary structure of MAPK proteins in Tartary buckwheat} The tertiary structures of MAPK proteins are shown in Fig. 5 , and they are mainly composed of $\alpha$-helices, $\beta$-folds and random coils. MAPK1, MAPK2, MAPK3, MAPK4,
MAPK5, MAPK6, MAPK7, MAPK8 and MAPK12 have identical structures, indicating that they have similar functions. In addition, MAPK9, MAPK10, MAPK13 and MAPK16 are structurally similar, as are MAPK11, MAPK14 and MAPK15 (Fig. 5). It is speculated that proteins with different structures may determine the diversity of FtMAPK functions.

\section{Gene duplication and synteny analysis of the FtMAPK gene families}

There are three pairs of segmental duplications on the chromosomes of Tartary buckwheat, and they are present only on chromosomes 1 and 8 (Fig. 6A). We speculate that the degree of evolution of FtMAPKs is relatively conservative within the species. To further understand the replication event of the FtMAPK genes, the replication events of $M A P K$ were compared between Tartary buckwheat and other species (Arabidopsis thaliana and Gossypium raimondii). These results also demonstrated that segmental duplication $M A P K$ gene pairs were found in the genomes of Arabidopsis (9 pairs) and Gossypium raimondii (19 pairs) (Fig. 6B-C).

The synteny between species provides insight for studying the evolution of gene families and gene function. Therefore, we analysed the collinearity of $M A P K$ between Tartary buckwheat and six other plants (Fig. 7). Collinearity analysis showed that the homologous genes between Fagopyrum tataricum and Glycine max were the most common, with 17 homologous gene pairs, followed by Vitis vinifera (11 homologous gene pairs), Solanum lycopersicum (11 homologous gene pairs), Arabidopsis thaliana (8 homologous gene pairs), Helianthus annuus 


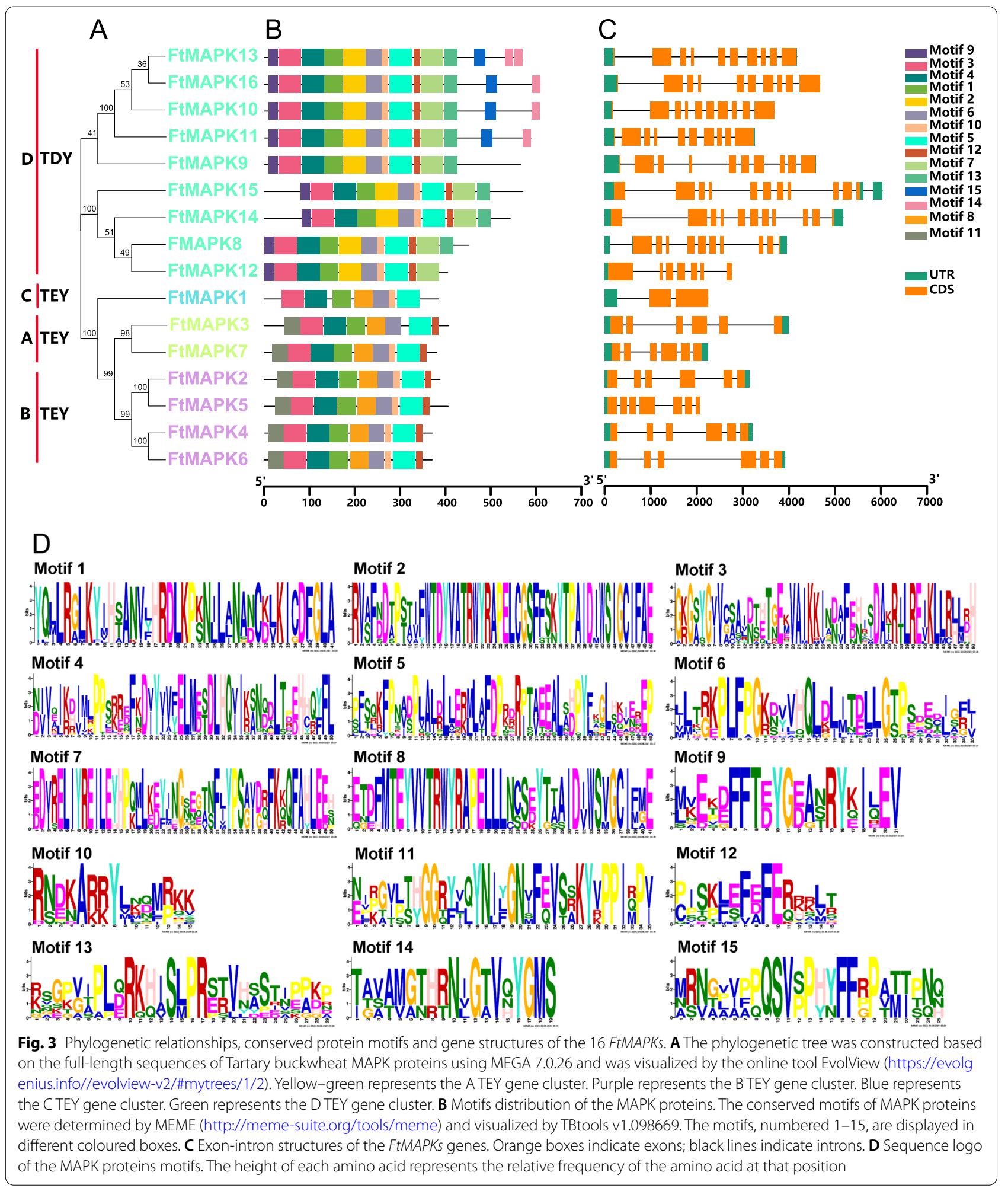

(7 homologous gene pairs) and Beta vulgaris (6 homologous gene pairs). In the collinearity analysis between Tartary buckwheat and soybean, FtMAPK2 had homologous pairs with five $M A P K$ genes in soybean, indicating that FtMAPK2 may play an essential role in the evolution of the Tartary buckwheat MAPK family. These results 

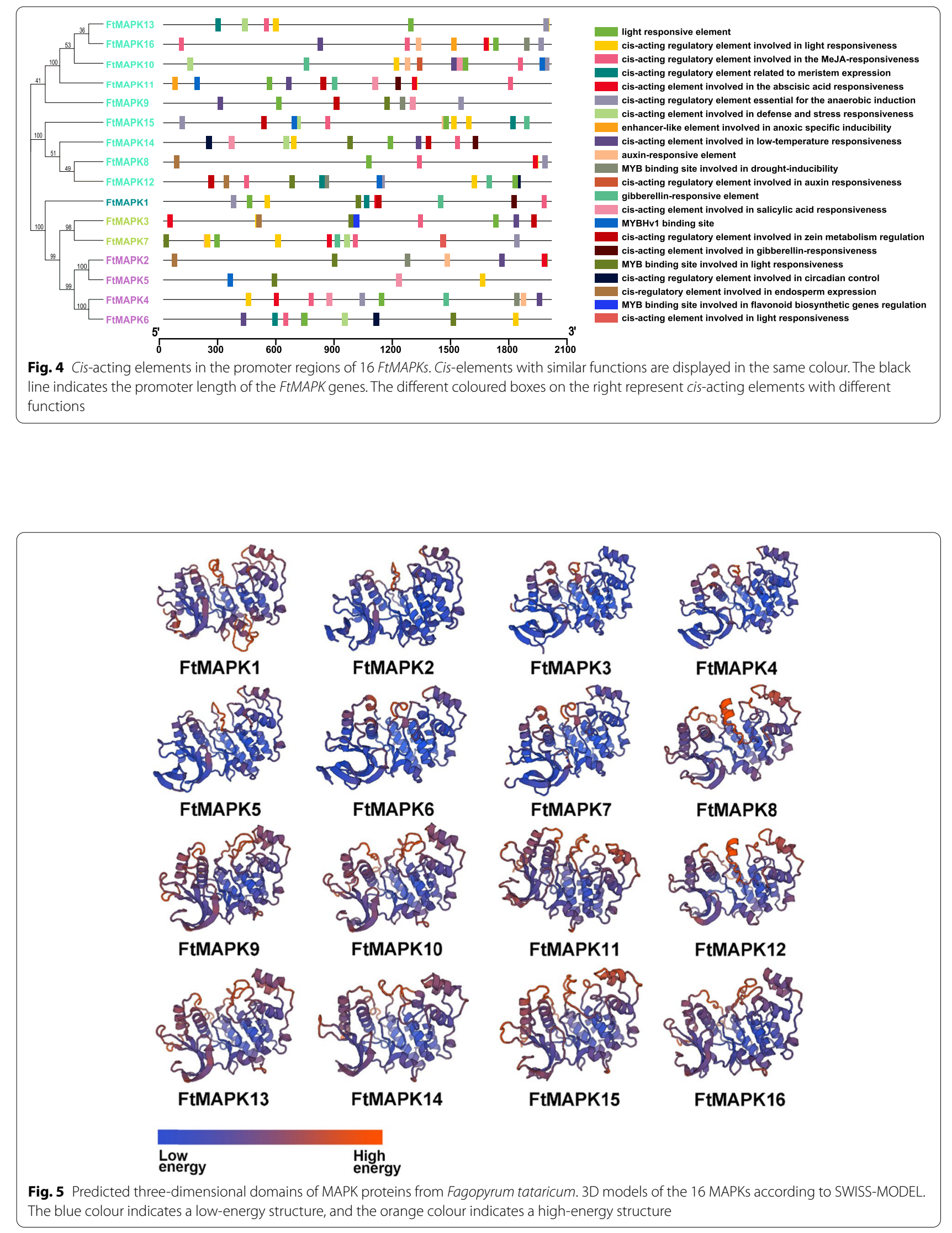


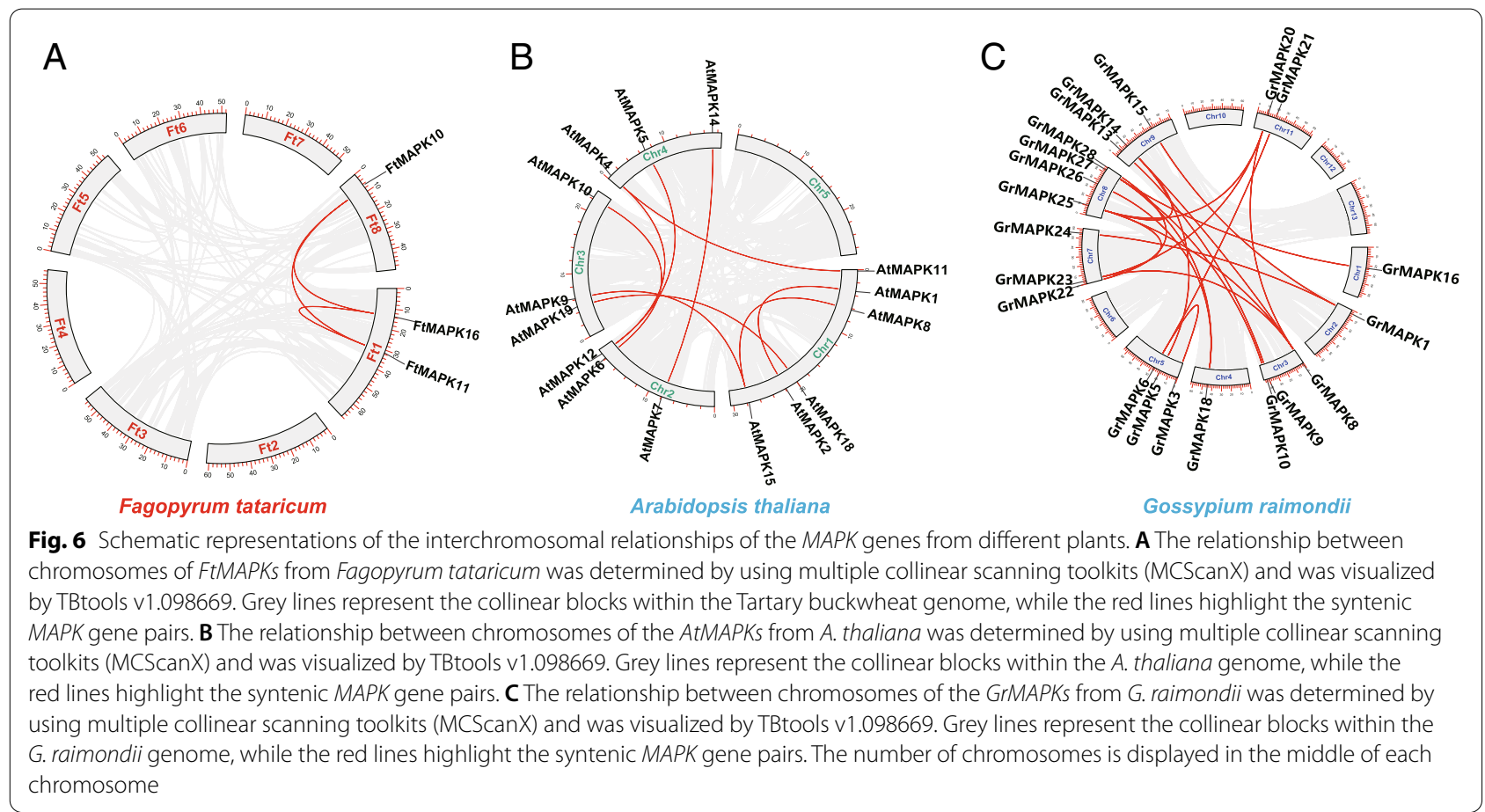

revealed that the correlation between FtMAPK genes and $G m M A P K$ genes is similar, which is significant for exploring the relationships among species and forecasting gene function.

\section{Expression analysis of FtMAPK genes in different tissues}

Exploring tissue-specific expression profiles provides a basis for studying gene functions. Therefore, we investigated the tissue-specific expression of FtMAPKs. The results showed that the expression levels of 16 FtMAPK genes in the roots, stems, leaves and flowers of Tartary buckwheat varied widely (Fig. 8). For instance, most FtMAPKs are ubiquitously expressed in every tissue of Tartary buckwheat, but FtMAPK12 is expressed only in leaves and flowers. FtMAPK3 had the highest expression in stems and leaves, and FtMAPK5 was predominantly expressed in roots, leaves and flowers compared with the other tissues; in contrast, FtMAPK4, FtMAPK6, FtMAPK7, FtMAPK9, FtMAPK11, FtMAPK13 and FtMAPK15 had lower expression levels in all tissues (Fig. 8).

\section{Expression of FtMAPK genes under different stress and hormone treatments}

Analysis of cis-acting elements indicated that most of the Pro ${ }_{\text {FtMAPKs }}$ contained MeJA and ABA response elements. We randomly selected a FtMAPK from each subfamily. To preliminarily investigate the potential role of MAPKs under hormone treatment (MeJA and $\mathrm{ABA}$ ), we determined the expression pattern of FtMAPKs by qRT-PCR (Fig. 9). Interestingly, the transcript levels of FtMAPK1 were upregulated in response to hormone processing and reached the highest levels at $48 \mathrm{~h}$. However, the above two hormone treatments downregulated the expression of the other three genes (FtMAPK3, FtMAPK4 and FtMAPK9) compared with their expression before treatment. ABA treatment induced FtMAPK1 and FtMAPK9 to reach their highest levels at $48 \mathrm{~h}$ and $3 \mathrm{~h}$, respectively. Compared with before treatment, the expression of FtMAPK3 and FtMAPK4 was downregulated.

We also investigated the role of FtMAPKs in the response to drought and salt stress (Fig. 10). The expression level of FtMAPK1 was significantly upregulated throughout the treatment process and reached its highest level at $48 \mathrm{~h}$, whereas the FtMAPK3, FtMAPK4 and FtMAPK9 genes were downregulated after salt treatment. With drought treatment, the expression levels of FtMAPK1 and FtMAPK3 were significantly upregulated and peaked at $12 \mathrm{~h}$ and $3 \mathrm{~h}$, respectively. However, the expression levels of FtMAPK4 and FtMAPK9 were downregulated at all treatment time points (Fig. 10). The downregulation of gene expression seems to indicate that the activation of certain protein kinase activities may not be related to their transcriptional levels under stress. 


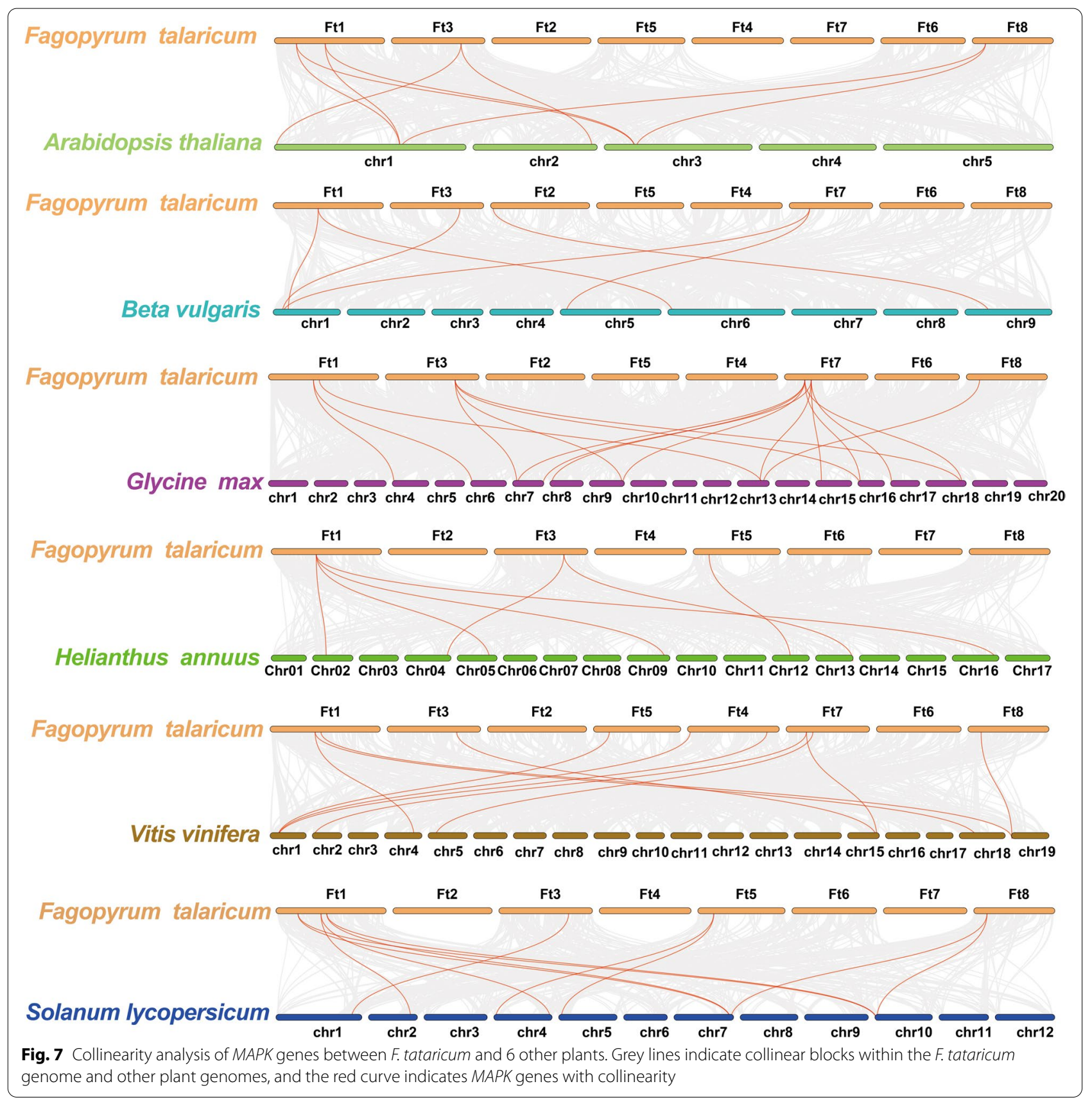

\section{Prediction and correlation analysis of MAPK interacting proteins}

The construction of protein interaction networks is significant for studying gene interactions and regulatory relationships. Here, to identify proteins that interact with MAPK in Tartary buckwheat, an interactive network involving MAPK1-9 and MAPK12-14 was created. The results show that MAPK5 can interact with WRKY74. Therefore, we speculate that MAPK5 regulates the expression of FtWRKY74 and ultimately responds to external stress (Fig. 11A). In addition, the interactive protein FtWRKY6 involved in the FtMAPK2 network can respond to salt, drought, cold and heat stress (Fig. 11B). FtAG and FtPISTILLATA are involved in the development of shelling and floral organs. We speculated that the interactive proteins AG and PISTILLATA-like participate in the MAPK3 network and regulate the development of Tartary buckwheat by interacting with MAPK3 (Fig. 11C). Additionally, FtMAPK12 regulates the expression of FtHY5-like (Fig. 11D). It has been reported that 

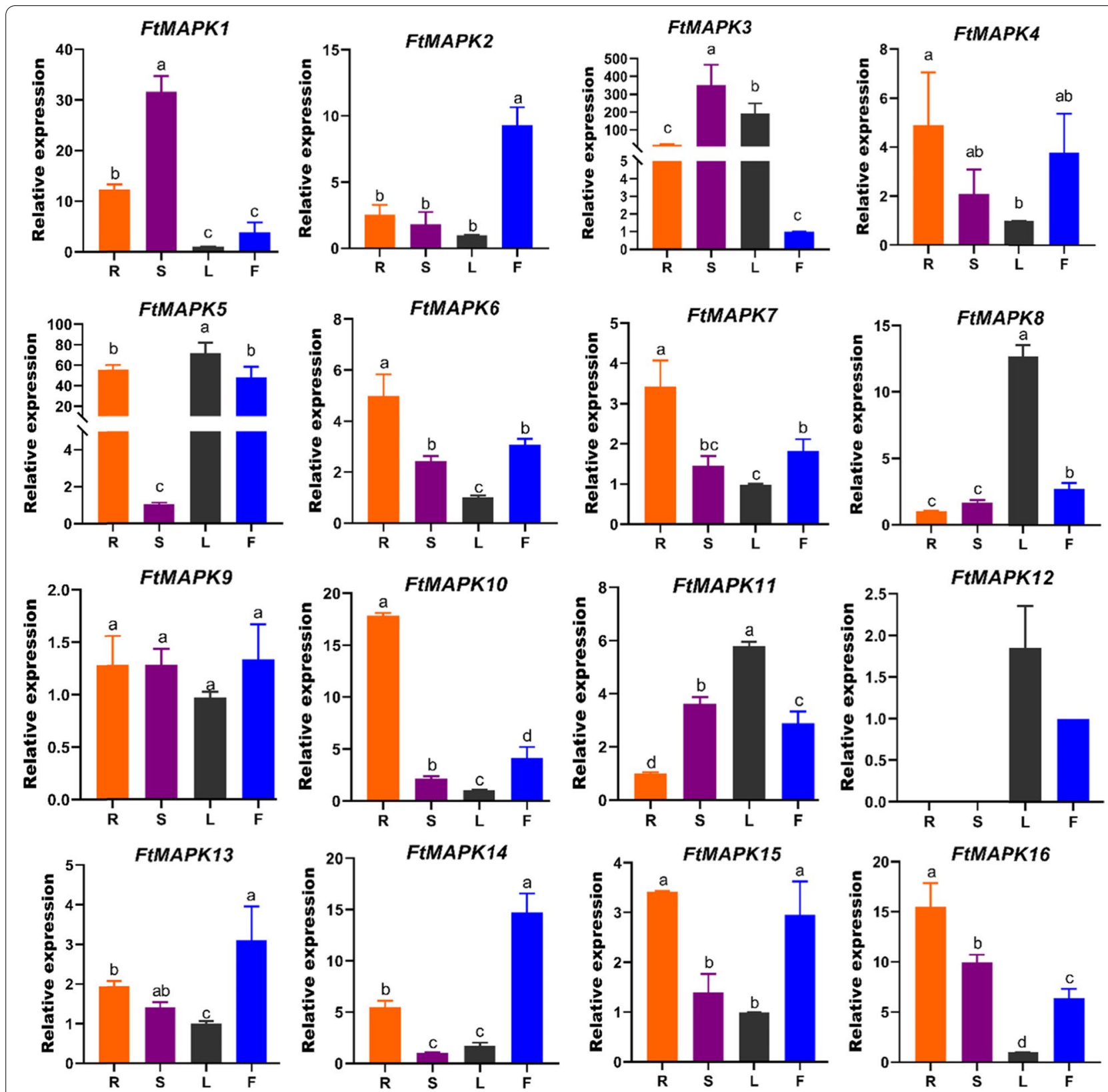

Fig. 8 qRT-PCR analysis of FtMAPKs in different tissues. R (root), S (stem), L (leaf), F (flower). The lowest expression level of all genes in the four tissues was set as 1 . The error bars were obtained from three measurements. Letter(s) above the bar represent significant differences ( $a=0.05$, Duncan) among different tissues

FtHY5 not only participates in the biosynthesis of anthocyanins but also participates in plant growth and development and response to stress [16-21]. These results preliminarily indicate that FtMAPKs participate in growth and development and severe vital roles in abiotic stress in plants.

To further investigate whether FtMAPKs are involved in drought or salt stress, we analysed the correlation between FtMAPKs and other transcription factors involved in the abiotic stress response (Fig. 12). The results showed a positive correlation between the expression levels of FtMAPK1 and FtNAC8, FtNAC9, FtNAC4, FtNAC6, FtMYB21 and FtbHLH4. We further confirmed that FtMAPK1 is involved in the abiotic stress response process. The correlation between FtMAPK4 and other transcription factors was significantly reduced. FtMAPK4 was negatively correlated with the expression level of most transcription factors compared with the correlation 

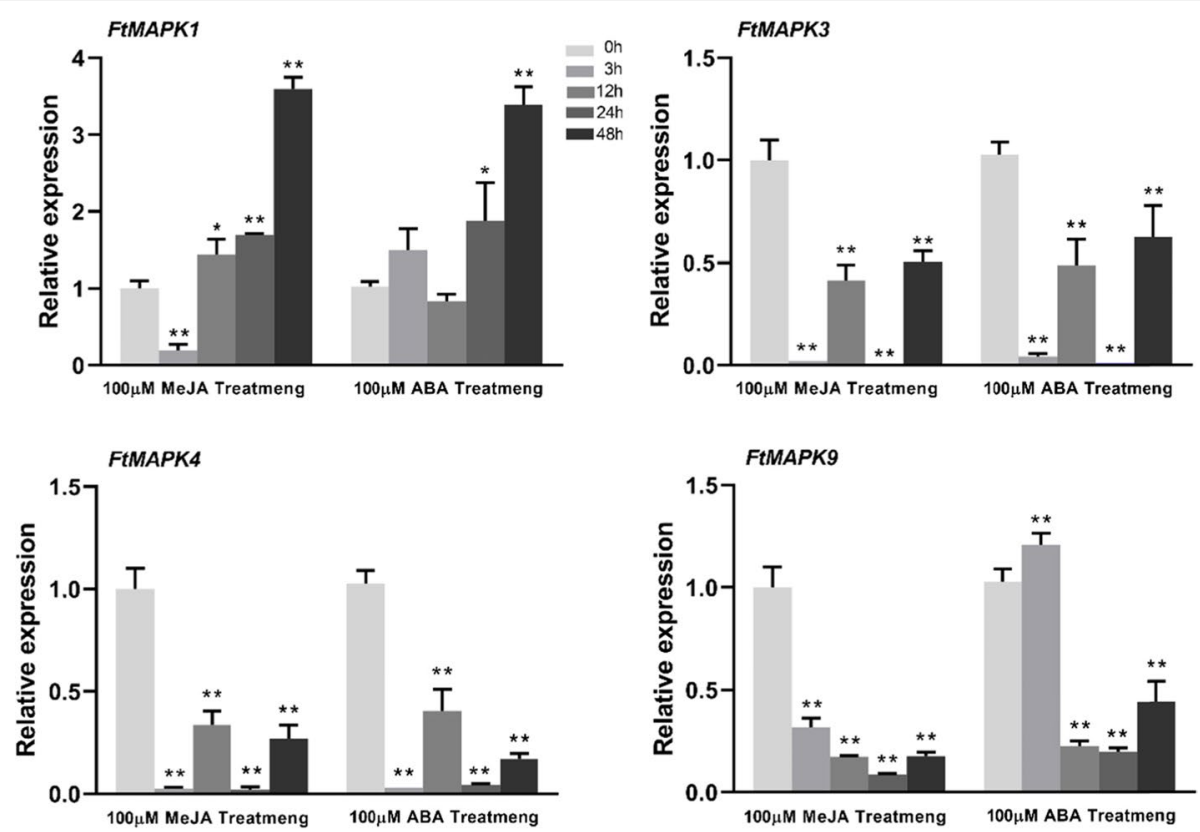

Fig. 9 Relative expression of FtMAPK genes under MeJA and ABA treatments. The expression of FtMAPK genes at $0 \mathrm{~h}$ in the dark was set to "1". The Values are the means \pm SDs $(n=3)$. The Bars represent the standard deviations of three independent experiments. The asterisks indicate significant differences $\left({ }^{*} P<0.05 ;{ }^{*} P<0.01\right)$
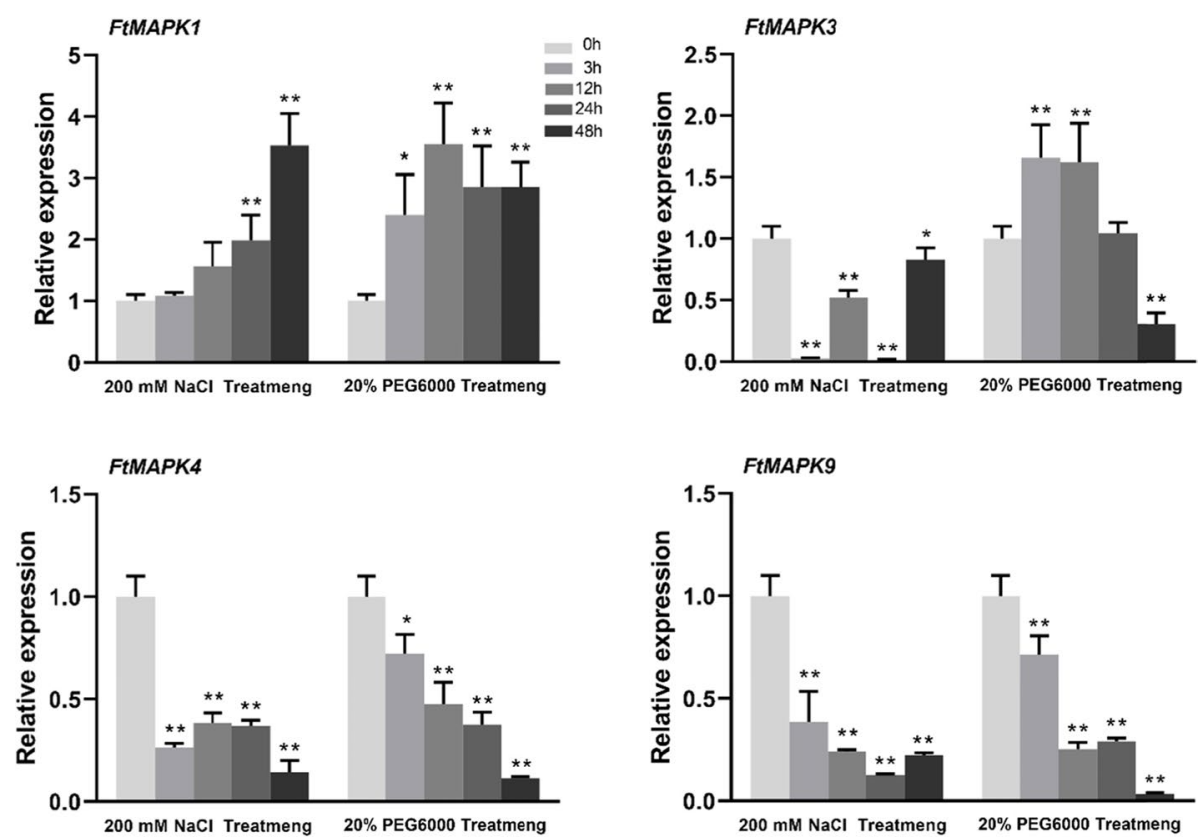

Fig. 10 Relative expression of FtMAPK genes under NaCl and PEG6000 treatments. The expression of FtMAPK genes at $0 \mathrm{~h}$ in the dark was set to "1". The bars represent the standard deviations of three independent experiments. The asterisks indicate significant differences $\left({ }^{*} P<0.05 ;{ }^{* *} P<0.01\right)$ 


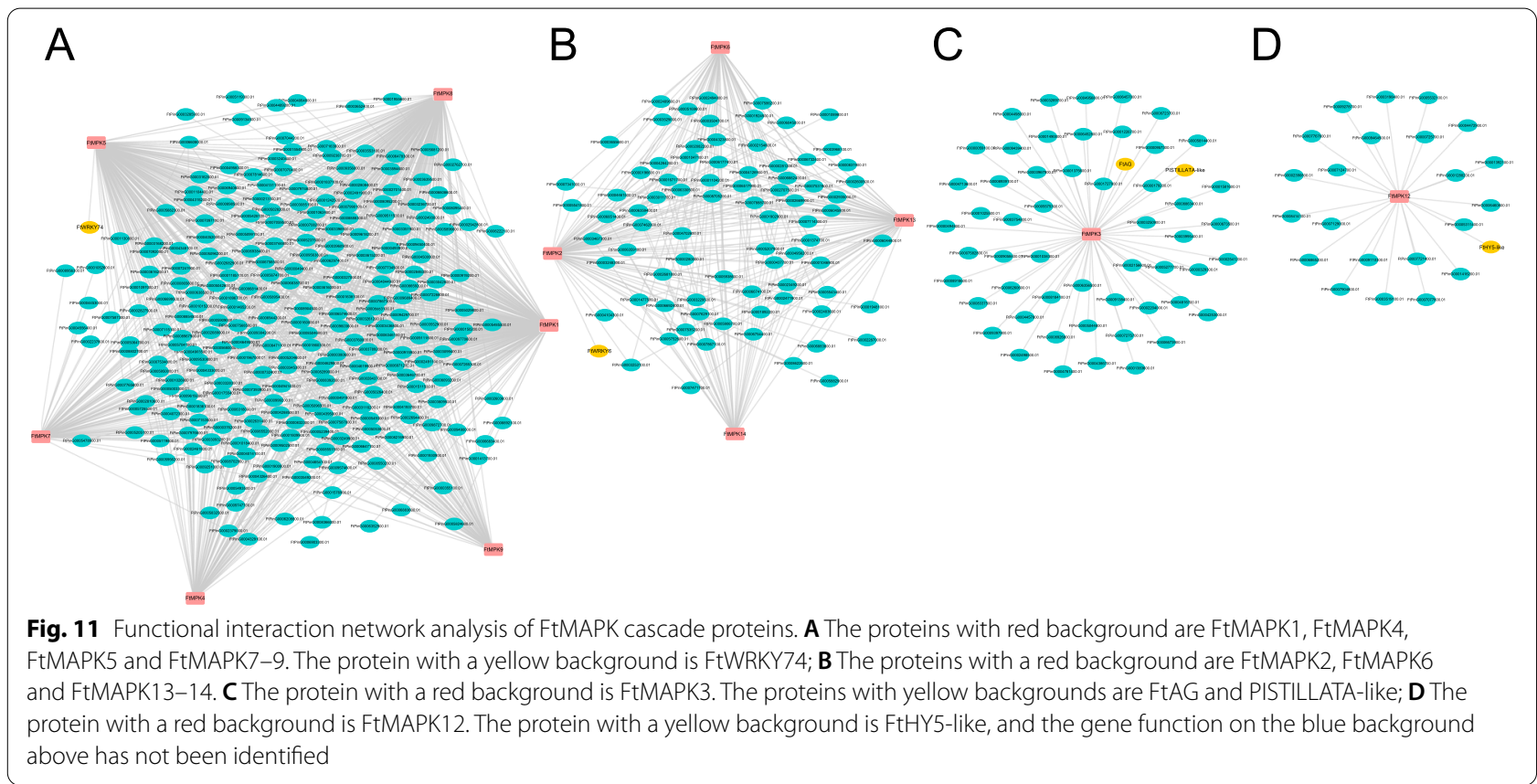

between FtMAPK1 and other transcription factors. There was a significant positive correlation among the expression levels of FtMAPK9, FtbHLH1 and FtMYB17. These results show that FtMAPK9 has the same expression pattern as FtbHLH1 and FtMYB17. In addition, there was a positive correlation among four FtMAPKs (FtMAPK2, FtMAPK6, FtMAPK8 and FtMAPK13) with respect to FtNAC2 and FtMYB9. There was a significant positive correlation between FtMAPK10 with respect to FtMYB10, FtMYB13 and FtNAC5. There was a significant positive correlation between FtMAPK11 and FtbHLH13. FtMAPK15, FtMAPK7 and FtMAPK9 had significant positive correlations with FtbHLH1 and FtMYB17. In summary, the correlation analysis shows that most FtMAPKs participate in the stress response.

\section{Effect of overexpression of FtMAPK1 on salt tolerance of Tartary buckwheat}

To determine the effect of FtMAPK1 overexpression on plant response to salt stress, 4-week-old Tartary buckwheat leaves were tested under the same growth conditions (Fig. 13A). Three days after transient transformation FtMAPK1, the expression level of FtMAPK1 was 1.8 times that of the control group (Fig. 13B). Antioxidant enzymes activity determination showed that the content of SOD and POD in the experimental group were significantly higher than those in the control group (Fig. 13C-D). It indicated that overexpression of FtMAPK1 enhanced the tolerance of Tartary buckwheat to salt stress.
To further study the mechanism by which FtMAPK1 regulates the downstream stress-related genes at the transcriptional level, the expression of four stress responsive genes were measured by qPCR (Fig. 13E). The results of the study showed that after salt treatment for 24 hours, the expression levels of FtMYB21, FtNAC4, FtNAC6 and FtNAC9 in the experimental group were significantly induced compared with the control group. Our results suggest that FtMAPK1 may enhance the resistance to salt stress by increasing downstream stress-related gene expression.

\section{Discussion}

The expansion of $M A P K$ family members is indispensable to plant evolution. Compared with the number of genes in other gene families, the amounts of the $M A P K$ gene family are relatively conserved in terms of evolution, but with the identification of $M A P K$ an increasing number of plants, we found that the number of $M A P K$ genes has also expanded. For example, 16, 16, 17, 21, 25 and 26 MAPK genes were identified in Brachypodium distachyon [22], tomato [23], tobacco [24], poplar [25], banana and apple [26],respectively, while there were 38 in soybean [27]. Legumes and woody species have more $M A P K$ genes. We speculate that leguminous and woody plant species may require more $M A P K$ genes to participate in their complex transcriptional regulation. To date, we screened only 16 FtMAPK genes in the genome of Fagopyrum tataricum compared with the number of $M A P K$ genes in soybean (38 members), 


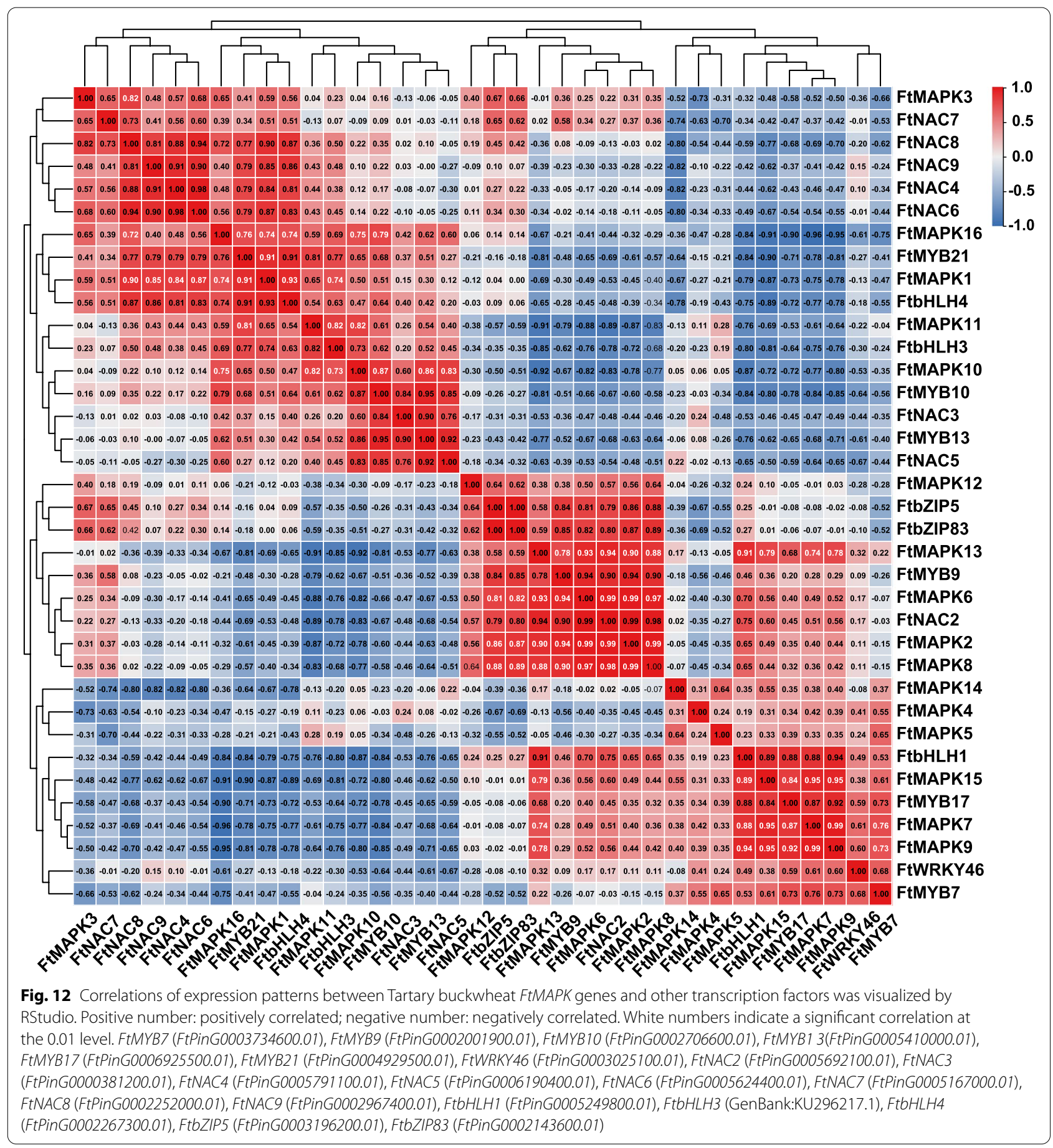

cotton (28 members) and bread wheat (54 members) (Fig. 1).

The number of $M A P K$ gene family members is related to genome size, replication events, and ploidy [28]. Higher plants have more $M A P K$ genes than lower plants. However, there are $54 M A P K$ genes in the bread wheat genome. Therefore, many $M A P K$ genes are related to their polyploidy in bread wheat [29]. Therefore, we speculate that genome duplication and plant evolution are essential for expanding $M A P K$ gene family members.

Researchers analysing the $M A P K$ genes of 40 species found that $M A P K$ s can be divided into six different subfamilies (A, B, C, D, E, and F) [28]. LIAN et al. [30] conducted phylogenetic tree analysis on 20 AtMPKs, 


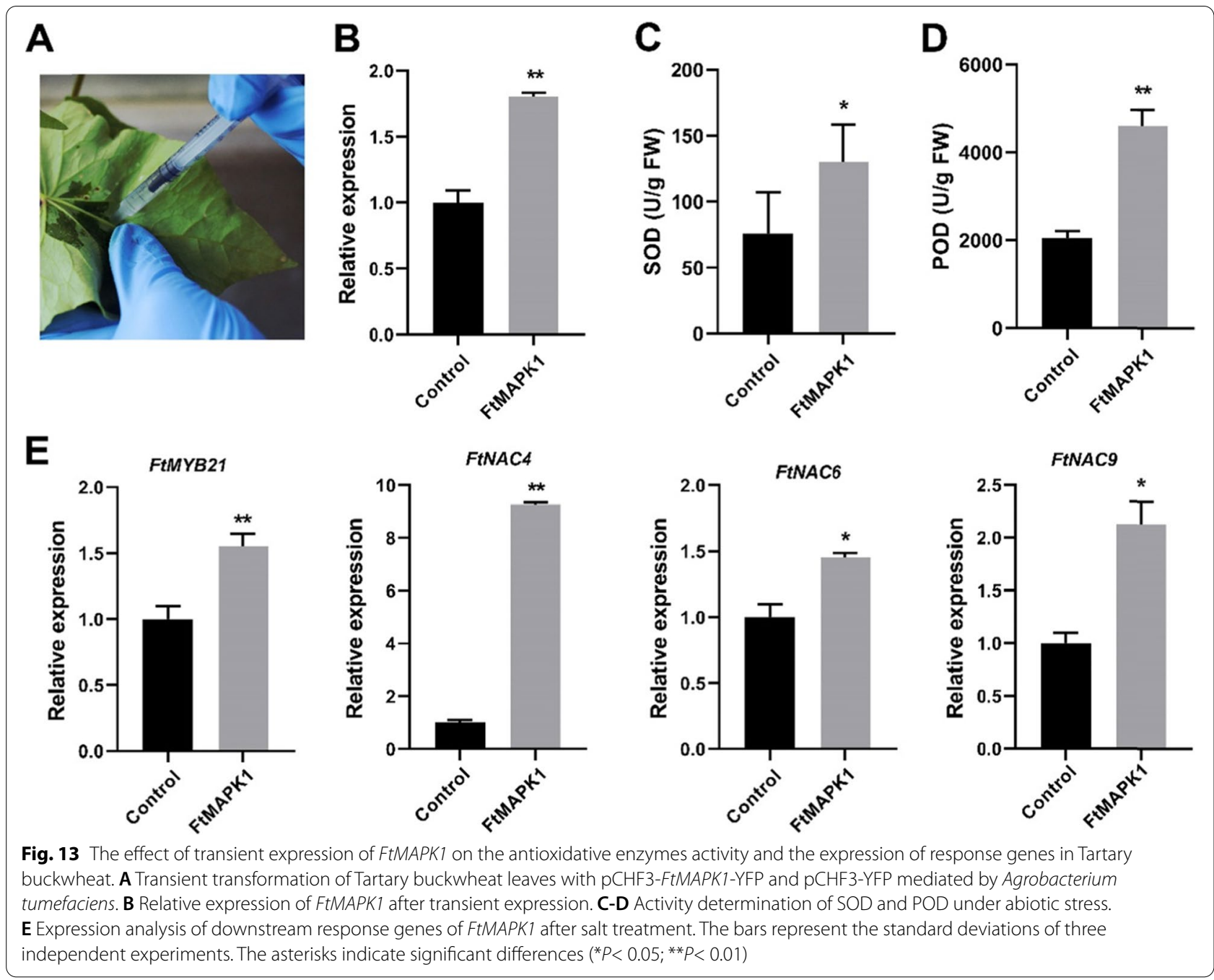

15TaMAPKs and 17 OsMPKs, and classified them into seven groups (A, B, C, D, E, F, and G). In this study, the A, B, C and D subfamilies containing TEY/TDY conserved motifs were consistent with those previously reported (Fig. 3B), and there were no $\mathrm{E}$ or $\mathrm{F}$ subfamilies. However, the two subfamilies $\mathrm{E}$ and $\mathrm{F}$ are shared by lower eukaryotes and gymnosperms, and we speculate that this may also be a reason for the expansion of gene family members. The emergence of new motifs in MAPK led to an expanded diversity of MAPKs. MAPK not only contains TEY/TDY motifs but also contain characteristic motifs such as MEY, TEM and TQY. The diversification of distinctive motifs provides ideas for investigating the function of $M A P K \mathrm{~s}$, and the diversification of functions enables plants to enhance their ability to adapt to the environment. Here, the 16 FtMAPKs contained only TEY/TDY characteristic motifs. This result also shows that the motifs in MAPK in Tartary buckwheat are relatively conserved. The increase in the number of $M A P K$ subfamilies and the diversification of characteristic motifs provide new ideas for understanding the evolution and gene function of the $M A P K$ gene family.

Promoters control the changes in their structure and morphology in different stages of plant growth and development and the interaction between plants and the environment. Throughout the long process of biological evolution, plants have acquired complex gene regulation mechanisms to mitigate the effects of unfavourable environments. $M A P K$ promoters include stress-related cis-acting elements, including ABA-responsive cis-acting (ABREs) and LTRs in chickpea [6]. In addition, the above mentioned cis-acting elements were found in the promoters of the MAPK genes of Solanum lycopersicum [23], B. distachyon [22] and cucumber [31], and the expression level of $M A P K s$ was changed after stress treatment. In our research, most FtMAPKs have cis-regulatory elements involved in ABA and LTR responses. Nevertheless, it is worth noting that 5 FtMAPKs (FtMAPK2, 
FtMAPK4, FtMAPK9, FtMAPK12 and FtMAPK16) contain MYB-binding sites involved in drought-induced responses, and 3 FtMAPKs (FtMAPK3, FtMAPK10 and FtMAPK16) can bind to MYB-binding sites to regulate the expression of flavonoid synthesis genes. Therefore, in-depth research on the structure, function and expression pattern of promoters will help clarify the regulatory mechanism at the transcriptional level of gene expression and enhance understanding of the life process. However, based on the current research status of promoters, the regulatory mechanisms of most promoters at the transcriptional level remain unclear. In the future, it will be necessary to explore the active sites of promoter-related elements through biological experimental techniques (such as CRISPR/Cas9) to study the transcriptional regulation mechanism of downstream target genes.

The $M A P K$ cascade signalling pathway is a conserved protein kinase cascade signalling pathway ubiquitous in eukaryotes $[32,33]$. Many research results show that the plant $M A P K$ cascade signalling pathway can be widely involved in various response signalling pathways such as those pertaining to drought, high salt and other abiotic stresses, various plant hormones and other signalling molecules, and growth and development [34, 35]. MPK6 is involved in the environmental response process. Under cold treatment, it can enhance cold tolerance and allow plants to survive [36]. After several drought stress treatments were applied to peanut plants, it was found that the expression of $M A P K \mathrm{~s}$ was upregulated in the $M A P K$ signalling pathway [37]. In this study, the transcription of FtMAPK1 was upregulated under hormone, drought and salt treatment, which suggested that this gene might also have important biological functions in abiotic stress and hormone signalling. Notably, the expression level of FtMAPK4 was downregulated under the above treatment, and it is speculated that the activation of MAPK4 protein kinase activity may not be related to its transcription level.

In this study, FtMAPK1 and FtMAPK3 were highly expressed in the roots, stems and leaves; FtMAPK5 had higher expression levels in the roots, leaves and flowers; and FtMAPK8 and FtMAPK10 were highly expressed in the leaves and roots (Fig. 8). In Camellia sinensis, TEA006436.1 is highly expressed in the apical buds, flowers, fruits, mature leaves and stems [38]. In Gossypium raimondii, GrMAPK27 has the highest transcription level in the leaves, and GrMAPK16 is expressed in all tissues (roots, stems, leaves and petals) and organs (anthers, ovules and fibres) [9].

$M A P K$ genes also present tissue-specific expression patterns. In Triticum aestivum, TaMAPK12 and TaMAPK15 are expressed only in the roots and flowers, respectively [30]. Compared with other tissues,
SIMAPK7 and SIMAPK12 are highly expressed in stamens [23]. Here, only FtMAPK12 shows tissue-specific expression in the leaves and flowers of Fagopyrum tataricum. FtMAPK1 and FtMAPK3 are remarkedly highly expressed in the stems. This indicates that these genes play potential roles in developing leaves and stems in Fagopyrum tataricum. These results provide ideas for further research on the biological functions of MAPK genes.

The molecular mechanism of plant stress resistance has been a hot topic in biological research. However, there are relatively few studies on the function of $M A P K \mathrm{~s}$ in response to adversity. It has been reported that $M A P K \mathrm{~s}$ can participate in abiotic stress responses. For instance, upregulated expression of tobacco $M A P K$ genes can improve plant resistance to abiotic stress [39]. Overexpression of OsMAPK5 can positively regulate the tolerance of plants to drought, salt and cold stress [40]. In this study, we aimed to fully understand the function of FtMAPK genes in Tartary buckwheat. Correlation analysis has a potential role in the investigation of gene function. We found that most FtMAPKs (FtMAPK1, FtMAPK2, FtMAPK6, FtMAPK7, FtMAPK8, FtMAPK9, FtMAPK10, FtMAPK11, FtMAPK13 and FtMAPK15) were positively correlated with one or more FtMYB, FtNAC, FtbZIP and FtWRKY involved in the stress response. We speculate that these FtMAPKs may have the same expression pattern as their significantly related transcription factors. This research provides information for research on FtMAPKs functions.

\section{Conclusion}

In this study, we identified 16 FtMAPK genes from the Tartary buckwheat genome. A phylogenetic tree was constructed showing that all of the MAPKs contain TDY and TEY characteristic motifs. Analysis of all MAPK protein motifs strongly supports this prediction. Correlation analysis showed that $M A P K 1$ and stress-related transcription factors have the same expression pattern. Additionally, a protein interaction network was constructed, which indicated that several MAPKs participate in the regulation of the expression of stress and development-related genes. Salt stress experiment showed that FtMAPK1 ultimately enhanced the stress resistance of Tartary buckwheat by increasing the activity of antioxidant enzymes and the expression of stress-related genes.

\section{Materials and methods}

\section{Identification of FtMAPK genes}

To screen the MAPK genes in Tartary buckwheat, we obtained genomic data from http://www.mbkba se.org/ Pinku1/. Arabidopsis MAPK cascade proteins from https:// www.arabidopsis.org/ were used as queries to search for Tartary buckwheat proteins using BLASTP. Candidate 
genes were submitted to https://www.ncbi.nlm.nih.gov/cdd to verify their characteristic kinase structural motifs further.

\section{Analysis of the main characteristics of MAPK family members in Tartary buckwheat}

The phylogenetic tree of $M A P K$ amino acid sequences in Tartary buckwheat and other three species (Arabidopsis thaliana, Oryza sativa and Gossypium raimondii) was constructed with MEGA 7.0.26 by the neighbour-joining (NJ) method and 1000 bootstrap replications, and the phylogenetic tree was visualized through EvolView (https://evolg enius.info//evolview-v2/\#mytrees/1/2). We analysed and visualized the distribution of all MAPKs on chromosomes of Tartary buckwheat by TBtools [41]. The phylogenetic tree of Tartary Buckwheat MAPK gene was constructed by NJ method and visualized by TBtools. The characteristic motifs of MAPKs were determined by MEME (http:// meme-suite.org/tools/meme), the number of motifs was 15 , and the order of site distribution was zero or one occurrence per sequence. TBtools analyses the gene structure of all MAPKs. In addition, to analyse cis-acting in the promoter region of the FtMAPK genes, we truncated the $2 \mathrm{~kb}$ sequence upstream of the start codon for FtMAPK genes, which was analysed via the PlantCARE database (http:// bioinformatics.psb.ugent.be/webtools/plantcare/html/). Finally, protein motifs and cis-acting elements were visualized by TBtools. The tertiary structure of FtMAPK proteins was predicted by SWISS-MODEL Interactive Workshop (https://www.swissmodel.expasy.org/interactive).

\section{Analysis of duplication events and collinearity of FtMAPKs}

The gene duplication events of Tartary buckwheat MAPK and collinearity analysis with six other species were assessed and performed, respectively, according to the method of Yao et al. [42].

\section{Plant growth, abiotic stress treatment}

The Tartary buckwheat cultivar 'Xiqiao 2' was grown at the experimental base of Sichuan Agricultural University in Ya'an, Sichuan, China, on May 10. For sampling times and sample storage conditions, refer to Yao et al. [42].

The cultivation of Tartary buckwheat seedlings was performed according to the method of Yuan et al. [43] with slight modifications. Hormonal $(100 \mu \mathrm{M}$ MeJA and 100 $\mu \mathrm{M}$ ABA ) and abiotic stress treatments $(200 \mathrm{mM} \mathrm{NaCl}$ and 20\% PEG6000 (w/v)) were performed according to the method of Zhang [9] and Singh [44]. The cotyledons were collected at $0,3,12,24$ and $48 \mathrm{~h}$, flash frozen in liquid nitrogen, and then stored at $-80^{\circ} \mathrm{C}$ for further analysis.

\section{Construction of a protein interaction network}

The MAPK protein sequence was aligned to String (http://string-db.org/) for protein-protein interaction prediction. The regulatory network of the other proteins and the MAPK proteins was visualized using Cytoscape (http://www.cytoscape.org/).

\section{Correlation analysis between MAPK and transcription factors}

To predict the function of FtMAPKs, functionally identified transcription factors, including MYB (FtMYB7, FtMYB9, FtMYB10, FtMYB13 and FtMYB17) [45], FtMYB21 [46], FtWRKY46 [47], NAC (FtNAC2, FtNAC3, FtNAC4, FtNAC5, FtNAC6, FtNAC7, FtNAC8 and FtNAC9) [48], bHLH (FtbHLH1 [49], FtbHLH3 [50] and FtbHLH4 [51]), FtbZIP5 [52] and FtbZIP83 [53] were used to analyse the expression pattern of FtMAPKs in Tartary buckwheat. Salt stress transcriptome data were obtained from the NCBI Sequence Read Archive (http:// www.ncbi.nlm.nih.gov/Traces/sra/) with the accession number PRJNA528524 [54]. Drought stress (20\% PEG6000) transcriptome data were generated in-house (unpublished). The correlation analysis between FtMAPK genes and transcription factors was calculated using the cor function in the $\mathrm{R}$ language with the default Pearson correlation coefficient.

\section{Transient expression of FtMAPK1 in Tartary buckwheat leaves}

To further study the function of the MAPK gene of Tartary buckwheat, we selected MAPK1 and cloned the complete coding sequence. The open reading frame (ORF) of FtMAPK1 was PCR-amplified using primers (Additional file 4). Then, the sequence was inserted into the vector, pCHF3-YFP with Clone Express (Vazyme, C112-02). The recombinant plasmid was mediated by Agrobacterium tumefaciens to transform Tartary buckwheat leaves. The empty vector pCHF3-YFP was transiently transformed under the same conditions as those used for the negative control.

Three days after transformation, the leaves of the experimental group and the control group were placed on MS plates containing $200 \mathrm{mM} \mathrm{NaCl}$ for $24 \mathrm{~h}$, and then the superoxide dismutase (SOD) and peroxidase (POD) content of the experimental group and the control group were determined. SOD and POD were determined by the previous methods [55].

\section{Quantitative real-time PCR analysis}

Total RNA was extracted by an EASY spin Plant RNA Kit (Aidlab, China). First-strand cDNAs were synthesized using HiScript ${ }^{\circledR}$ III-RT SuperMix for qPCR (Vazyme, China). The qRT-PCR primers (Additional file 5) were designed via primer 3 (https://www.ncbi.nlm.nih.gov/ tools/primer-blast/). Refer to Liu et al. [56] for the selection of the internal reference genes. 2xChamQ Universal 
SYBR qPCR Master Mix (Vazyme, China) was used for qRT-PCR. The amplification program used were as follows: $98^{\circ} \mathrm{C}$ for $45 \mathrm{~s}$ followed by 34 cycles of $98^{\circ} \mathrm{C}$ for $15 \mathrm{~s}$ and $60{ }^{\circ} \mathrm{C}$ for $45 \mathrm{~s}$. The correlative expression data were calculated by the $2^{-\Delta \Delta \mathrm{CT}}$ method [57].

\section{Statistical analysis}

The experimental data were visualized by GraphPad Prism 8.0, and the significance was analyzed at the 0.05 and 0.01 probability levels by ANOVA in IBM SPSS Statistics 22.0.

\section{Abbreviations}

MAPK: Mitogen-activated protein kinase; Ft: Fagopyrum tataricum; MeJA: Methyl jasmonate; ABA: Abscisic acid.

\section{Supplementary Information}

The online version contains supplementary material available at https://doi. org/10.1186/s12864-022-08293-2.

Additional file 1 : Table S1. List of the 16 MAPK genes identified in this study

Additional file 2 : Figure S1. Analysis of characteristic domains of 16 FtMAPK proteins.

Additional file $\mathbf{3}$ : Table S2. Cis-acting elements of FtMAPKs promoter

Additional file 4 : Table S3. Primers used for construct expression vector of FtMAPK1. For expression of recombinant plasmid in Tartary buckwheat using PCHF-YFP vector.

Additional file 5 : Table S4. primer sequences of RT-qPCR

\section{Acknowledgments}

The authors are grateful to all colleagues in our laboratory for very useful discussions and technical assistance. We would like to thank the editor and reviewers for their constructive comments, valuable recommendations, and feedback, which facilitated the quality of article.

\section{Authors' contributions}

Yingjun Yao and Qi Wu designed the experiments, performed most of the experiments and wrote the manuscript. Haixia Zhao and Lei Sun performed the qRT-PCR. Wenjing Wu and Chenglei Li consulted the literature and analysed the data. All authors have read and approved the final manuscript.

\section{Funding}

This research was supported by the National Natural Science Foundation of China (31871699).

\section{Availability of data and materials}

The FtMAPK gene sequences in this study are available in the Tartary Buckwheat Genome Project (http://www.mbkbase.org/Pinku1/). The Tartary buckwheat cultivar 'Xiqiao 2' materials used in the experiment came were obtained our laboratory. The datasets supporting the conclusions of this article are included with in this article and its additional files.

\section{Declarations}

\section{Ethics approval and consent to participate}

The Tartary buckwheat cultivar 'Xiqiao 2' materials used in the experiment were obtained from our laboratory. These plant materials are widely used worldwide, and no permits are required for the collection of plant samples. This article did not contain any studies with human participants or animals and did not involve any endangered or protected species.
Consent for publication

Not applicable.

\section{Competing interests}

The authors declare that they have no competing interests.

Received: 20 Auqust 2021 Accepted: 3 January 2022

Published online: 03 February 2022

\section{References}

1. Cristina Suarez Rodriguez M, Petersen M, Mundy J. Mitogen-activated protein kinase signaling in plants. Annu Rev Plant Biol. 2010;611:621-49.

2. Colcombet J, Hirt H. Arabidopsis MAPKs: a complex signalling network involved in multiple biological processes. Biochem J. 2008;413:217-26.

3. Ichimura K, Shinozaki K, Tena G, Walker JC. Mitogen-activated protein kinase cascades in plants: a new nomenclature. Trends Plant Sci. 2002;77:301-8

4. Chen J, Wang LH, Yuan M. Update on the roles of rice MAPK cascades. Int J Mol Sci. 2021;224:1679.

5. Liu YK, Zhang D, Wang L, Li DQ. Genome-wide analysis of mitogenactivated protein kinase gene family in maize. Plant Mol Biol Report. 2013:316:1446-60.

6. Singh A, Nath O, Singh S, Kumar S, Singh IK. Genome-wide identification of the MAPK gene family in chickpea and expression analysis during development and stress response. Plant Gene. 2017;13:25-35.

7. Wang G, Wang T, Jia Z-H, Xuan J-P, Pan D-L, Guo Z-R, et al. Genome-wide bioinformatics analysis of MAPK gene family in Kiwifruit (Actinidia Chinensis). Int J Mol Sci. 2018;19:2510.

8. Cui LC, Yang G, Yan JL, Pan Y, Nie XJ. Genome-wide identification, expression profiles and regulatory network of MAPK cascade gene family in barley. BMC Genomics. 2019;20:750.

9. Zhang $X Y$, Wang $L M, X u X Y$, Cai $C P$, Guo WZ. Genome-wide identification of mitogen-activated protein kinase gene family in Gossypium raimondii and the function of their corresponding orthologs in tetraploid cultivated cotton. BMC Plant Biol. 2014;14:345.

10. Zhan HS, Hong Y, Zhao X, Wang M, Song WN, Nie XJ. Genome-wide identification and analysis of MAPK and MAPKK gene families in bread wheat (Triticum aestivum L.). Genes. 2017:8:284.

11. Zhou HY, Ren SY, Han YF, Zhang Q, Qin L, Xing Y. Identification and analysis of mitogen-activated protein kinase (MAPK) cascades in Fragaria vesca. Int J Mol Sci. 2017;18:1766.

12. Lee SK, Kim BG, Kwon TR, Jeong MJ, Park SC. Overexpression of the mitogen-activated protein kinase gene OSMAPK33 enhances sensitivity to salt stress in rice (Oryza sativa L.). J Biosci. 2011:361:139-51.

13. Bush SM, Krysan PJ. Mutational evidence that the Arabidopsis MAP kinase MPK6 is involved in anther, inflorescence, and embryo development. J Exp Bot. 2007;58:2181-91.

14. Huang $K$, Wu T, Ma ZM, Li Z, Chen HY, Zhang MX, et al. Rice transcription factor OsWRKY55 is involved in the drought response and regulation of plant growth. Int J Mol Sci. 2021;22:4337.

15. Yang T, Ma HY, Li Y, Zhang Y, Zhang J, Wu T, et al. Apple MPK4 mediates phosphorylation of MYB1 to enhance light-induced anthocyanin accumulation. Plant J. 2021;106:1728-45.

16. Lee $S$, Wang WL, Enamul H. Spatial regulation of thermomorphogenesis by HY5 and PIF4 in Arabidopsis. Nat Commun. 2021;12:3656.

17. Gao YQ, Bu LH, Han ML, Wang YL, Li ZY, Liu HT, et al. Long-distance blue light signalling regulates phosphate deficiency-induced primary root growth inhibition. Mol Plant. 2021;14:1539-53.

18. Li S, Chen KS, Donald G. Molecular and hormonal mechanisms regulating fleshy fruit ripening. Cells. 2021;10:1136.

19. Truong HA, Lee S, Trịnh CS, Lee WJ, Chung E-H, Hong S-W, et al. Overexpression of the HDA15 gene confers resistance to salt stress by the induction of NCED3, an ABA biosynthesis enzyme. Front Plant Sci. 2021;12:640443.

20. Zhang LY, Jiang XC, Liu QY, Golam JA, Lin R, Wang LY, et al. The HY5 and MYB15 transcription factors positively regulate cold tolerance in tomato via the CBF pathway. Plant Cell Environ. 2020;43:2712-26.

21. An JP, Qu FJ, Yao JF, Wang XN, You CX, Wang XF, et al. The bZIP transcription factor MdHY5 regulates anthocyanin accumulation and nitrate assimilation in apple. Hortic Res. 2017;4:17056. 
22. Chen LH, Hu W, Tan SD, Wang M, Ma ZB, Zhou SY, et al. Genome-wide identification and analysis of MAPK and MAPKK gene families in brachypodium distachyon. PLoS One. 2012;7:1-19.

23. Kong FL, Wang J, Cheng L, Liu SY, Wu J, Peng Z, et al. Genome-wide analysis of the mitogen-activated protein kinase gene family in Solanum lycopersicum. Gene. 2012;499:108-20.

24. Zhang XT, Cheng TC, Wang GH, Yan YF, Xia QY. Cloning and evolutionary analysis of tobacco MAPK gene family. Mol Biol Rep. 2012;402:1407-15.

25. Nicole MC, Hamel LP, Morency MJ, Beaudoin N, Ellis BE, Séguin A. MAPping genomic organization and organ-specific expression profiles of poplar MAP kinases and MAP kinase kinases. BMC Genomics. 2006;71:223.

26. Zhang SZ, Xu RR, Luo XC, Jiang ZS, Shu HR. Genome-wide identification and expression analysis of MAPK and MAPKK gene family in Malus domestica. Gene. 2013:531:377-87.

27. Neupane A, P.Nepal M, Piya S, Subramanian S, Rohila J, Reese R.N, et al. Identification, nomenclature, and evolutionary relationships of mitogenactivated protein kinase (MAPK) genes in soybean. Evol Bioinforma. 2013;99:363-86.

28. Kumar Mohanta T, Kumar Arora P, Mohanta N, Parida P, Bae H. Identification of new members of the MAPK gene family in plants shows diverse conserved domains and novel activation loop variants. BMC Genomics. 2015;16:58.

29. Kumar-De K, Saha A, Tamang R, Sharma B. Investigation on relative genome sizes and ploidy levels of Darjeeling-Himalayan Rhododendron species using flow cytometer. Indian J Biotechnol. 2010;91:64-8.

30. Lian W-W, Tang Y-M, Gao S-Q, Zhang Z, Zhao X, Zhao C-P. Phylogenetic analysis and expression patterns of the MAPK Gene family in wheat (Triticum aestivum L.). J Integr Agric. 2012;11:1227-35.

31. Wang J, Pan CT, Wang Y, Ye L, Wu J, Chen LF, et al. Genome-wide identification of MAPK, MAPKK, and MAPKKK gene families and transcriptional profiling analysis during development and stress response in cucumber. BMC Genomics. 2015;16:386.

32. Šamajová O, Komis G, Samaj J. Emerging topics in the cell biology of mitogen-activated protein kinases. Trends Plant Sci. 2013;183:140-8.

33. Šamajová O, Plíhal O, Al-Yousif M, Hirt H, Šamaj J. Improvement of stress tolerance in plants by genetic manipulation of mitogen-activated protein kinases. Biotechnol Adv. 2013;31:118-28.

34. Sinha AK, Jaggi M, Raghuram B, Tuteja N. Mitogen-activated protein kinase signaling in plants under abiotic stress. Plant Signal Behav. 2011;62:196-203.

35. Hirt H. Multiple roles of MAP kinases in plant signal transduction. Trends Plant Sci. 1997;2:11-5

36. Giordano Ponce-Pineda L, Carmona-Salazar L, Saucedo-García M, CanoRamírez D, Morales-Cedillo F, Peña-Moral A, et al. MPK6 kinase regulates plasma membrane H-ATPase activity in cold acclimation. Int J Mol Sci. 2021:22:6338.

37. Zhao NN, Cui SL, Li XK, Liu BK, Deng HT, Liu YR, et al. Transcriptome and co-expression network analyses reveal differential gene expression and pathways in response to severe drought stress in peanut ( Arachis hypogaea L.). Front Genet. 2021;12:672884.

38. Chatterjee A, Paul A, Unnati GM, Rajput R, Biswas T, Kar T, et al. MAPK cascade gene family in Camellia sinensis: In-silico identification, expression profiles and regulatory network analysis. BMC Genomics. 2020;21:1-17.

39. Wu H, Li HY, Zhang WH, Tang H, Yang L. Transcriptional regulation and functional analysis of Nicotiana tabacum under salt and ABA stress ScienceDirect. Biochem Biophys Res Commun. 2021;570:110-6.

40. Xiong LZ, Yang YN. Disease resistance and abiotic stress tolerance in rice are inversely modulated by an abscisic acid-inducible mtogen-activated protein kinase. Plant Cell. 2003;15:745-59.

41. Chen CJ, Chen H, Zhang Y, Hannah RT, Margaret HF, et al. TBtools: an integrative toolkit developed for interactive analyses of big biological data. Mol Plant. 2020;13:1194-202.

42. Yao YJ, Sun L, Wu WJ, Wang S, Wu Q, et al. Genome-wide investigation of major enzyme-encoding genes in the flavonoid metabolic pathway in Tartary buckwheat (Fagopyrum tataricum). J Mol Evol. 2021;89:269-86.

43. Yuan JP, Shen CW, Chen BH, Shen AM, Li XZ. Genome-wide characterization and expression analysis of CAMTA gene family under salt stress in Cucurbita moschata and Cucurbita maxima. Front Genet. 2021;12:647339.

44. Singh A, Nath O, Singh S, Kumar S, KSingh IK. Genome-wide identification of the MAPK gene family in chickpea and expression analysis during development and stress response. Plant Gene. 2017;13:25-35.
45. Gao F, Zhao HX, Yao HP, Li CL, Chen H, Wang AH, et al. Identification, isolation and expression analysis of eight stress-related R2R3-MYB genes in tartary buckwheat (Fagopyrum tataricum). Plant Cell Rep. 2016;356:1385-96.

46. Huang YJ, Deng RY, Gao F, Luo XP, Wu Q. Cloning and expression analysis of transcription factor gene FtMYB21 from Tartary buckwheat under abiotic stress (in Chinese). Genomics Appl Biol. 2015;34:1939-45.

47. Lv BB, Wu Q, Wang AH, Li Q, Dong QX, Kang JY, et al. A WRKY transcription factor, FtWRKY46, from Tartary buckwheat improves salt tolerance in transgenic Arabidopsis thaliana. Plant Physiol Biochem. 2020;147:43-53.

48. Deng RY, Zhao HX, Xiao YH, Huang YJ, Yao PF, Lei YL, et al. Cloning, characterization, and expression analysis of eight stress-related NAC genes in Tartary buckwheat. Crop Sci. 2019;591:266-79.

49. Luo XP, Zhao HX, Xue J, Li CL, Chen H, Park S-U, et al. Cloning of two basic helix-loop-helix transcription factor genes from Tartary buckwheat (Fagopyrum tataricum) and their expression under abiotic stress. Turk J Biol. 2016;40:1192-201.

50. Yao PF, Zhao XR, Li MF, Wang AH, Wu Q. Cloning and expression analysis of transcription factor gene FtHLH3 from Fagopyrum tataricum under abiotic stress (in Chinese). Genomics Appl Biol. 2016;35:429-35.

51. Zhou J, Gao F, Zhang RM, Luo XP, Huang Y, Li CL, et al. Cloning and expression of FtHLH4 in Fagopyrum tataricum and response to abiotic stress (in Chinese). Acta Bot Boreal. 2015;35:2399-404.

52. Li Q, Zhao HX, Wang XL, Kang JY, Wu Q. Tartary buckwheat transcription factor FtbZIP5, regulated by FtSnRK2.6, can improve salt/drought resistance in transgenic Arabidopsis. Int J Mol Sci. 2020;213:1123.

53. Li Q, Wu Q, Wang AH, Lv BB, Dong QX, Yao YY, et al. Tartary buckwheat transcription factor FtbZIP83 improves the drought/salt tolerance of Arabidopsis via an ABA-mediated pathway. Plant Physiol Biochem. 2019;144:312-23.

54. Ma WB, Jae KK, Jia CH, Yin FF, Hyo JK, Waheed A, et al. Comparative Transcriptome and Metabolic Profiling Analysis of Buckwheat (Fagopyrum Tataricum (L.) Gaertn.) under Salinity Stress. Metabolites. 2019;9:225.

55. O.Kotchoni S, Kuhns C, Ditzer A, Kirch H-H, Bartels D. Over-expression of different aldehyde dehydrogenase genes in Arabidopsis thaliana confers tolerance to abiotic stress and protects plants against lipid peroxidation and oxidative stress. Plant Cell Environ. 2006;29:1033-48.

56. Sun WJ, Yu HM, Liu MY, Ma ZT, Chen H. Evolutionary research on the expansin protein family during the plant transition to land provides new insights into the development of Tartary buckwheat fruit. BMC Genomics. 2021;22:252.

57. Livak KJ, Schmittgen TD. Analysis of relative gene expression data using real-time quantitative PCR. Methods. 2002;254:402-8.

\section{Publisher's Note}

Springer Nature remains neutral with regard to jurisdictional claims in published maps and institutional affiliations. 